\title{
The role of body mass index of husband and wife on the neonatal BMI and clinical outcome in ICSI
}

\begin{abstract}
Objective: To investigate the effects of body mass index (BMI) of husband and wife on the neonatal weight and clinical outcome in intracytoplasmic sperm injection (ICSI) cycles.
\end{abstract}

Methods: A Prospective cohort study that was conducted on patients undergoing ICSI cycle from February 2016 to March 2018.

Results: By investigating various factors by linear regression analysis only female BMI and GA at delivery ( $p$ value $<0.001$ ) had statistically significant increasing impact, while pregnancy number had statistically significant decreasing impact on neonatal birth weight ( $\mathrm{p}$ value $<0.001$ ). Only female obesity and multiple pregnancy had statistically significant raising effect ( $p$ values $=0.011,<0.001$ consecutively) whereas gestational age had statistical significant reducing impact on NICU admission ( $\mathrm{p}$ value $<0.001$ ).

Discussion: Also in the present study, studying different factors by linear regression only female BMI and GA at delivery $(p$ value $<0.001$ ) had significant increasing effects, while pregnancy number had statisyically significant decreasing effects on neonatal birth weight ( $\mathrm{p}$ value $<0.001$ )

Conclusion: Neonatal birth weight in ICSI is significantly influenced by raised maternal BMI and gestational age at wich delivery occurred.

Keywords: body mass index, intracytoplasmic sperm injection, neonatal BMI

\author{
Volume 9 Issue 5 - 2018 \\ Mahmoud Alalfy,' Ahmed Elgazzar, ${ }^{2}$ Omar \\ Abdelfattah,' Tamer Fares, ${ }^{3}$ Nevein Ghamry,' \\ Ahmed el lithy, ${ }^{4}$ Hasan Gaafar, ${ }^{4}$ Rana \\ Abdella, ${ }^{4}$ Mahmoud Soliman, ${ }^{5}$ A Hany, ${ }^{5}$ \\ Mohamed Hussein, ${ }^{5}$ Mohamed Elsharkawy, ${ }^{4}$ \\ IM Elzahaby, ${ }^{5}$ Mohamed Nabil, ${ }^{5}$ Rehab Lotfy, \\ Mohamed Ramadan, ${ }^{5}$ Dina Hatem, ${ }^{5}$ Ahmed \\ Hassan, ${ }^{6}$ Dalia Farouk, ${ }^{5}$ Alaa Hamed, ${ }^{7}$ Marwa \\ sharaf, ${ }^{5}$ Shimaa Mostafa Abd-El-Fatah, ${ }^{5}$ \\ Mohamed Ezz eldin, ${ }^{8}$ Ahmed Samy ${ }^{5}$ \\ 'Lecturer, Obstetrics and Gynecology, Department of \\ Reproductive Health and Family Planning, National Research \\ Centre, Egypt \\ 2Professor of Obstetrics and Gynecology, Cairo University, \\ Egypt \\ ${ }^{3}$ Assistant professor of Obstetrics and Gynecology, AlAzhar \\ University, Egypt \\ ${ }^{4}$ Assistant professor of Obstetrics and Gynecology, Cairo \\ University, Egypt \\ ${ }^{5}$ Lecturer of Obstetrics and Gynecology, Cairo University, Egypt \\ ${ }^{6}$ Lecturer of Obstetrics and Gynecology, Helwan University, \\ Egypt \\ ${ }^{7}$ Lecturer of Obstetrics and Gynecology,AlAzhar University, \\ Egypt \\ ${ }^{8}$ Specialist of Obstetrics and Gynecology,Aljazeerah Hospital, \\ Egypt
}

Correspondence: Mahmoud Alalfy, Lecturer, Department of Reproductive Health and Family Planning, National Research Centre, Egypt, Consultant, Aljazeerah Hospital, Giza, Egypt, Tel 010026II058,Email mahmoudalalfy@ymail.com

\section{Introduction}

There is increasing debate about the possible impact of parental obesity on the neonatal outcome and health. Body mass index is a crucial clinical feature for both planning the induction protocol and counseling for the probabilities of success after ICSI. ${ }^{1-3}$

Pre conception maternal obesity negatively impacts neonates, with a greater incidence of fetal macrososmia denoting that maternal BMI have an influence on neonatal BMI among other clinical parameters and features most research studies of BMI and ART outcomes have only investigated female or male BMIs. ${ }^{1-3}$

Few research studies have investigated the conjugated impact of couples' BMIs on ICSI outcomes.Prior research studies revealed that within the singleton gestations, BMI gains in couples was linked to raised neonatal birth weight and fetal macrosomia rates of singletons during IVF and ICSI cycles. ${ }^{1-3}$

Research efforts are required to reveal the impact of couples BMI levels on a spectrum of maternal and child clinical outcomes. ${ }^{4-6}$

Some research studies have displayed that mothers with higher body mass indices have poorer clinical outcomes than normal- BMI females undergoing ICSI, on the other hand another retrospective research study that recruited around 880 females as study subjects undergoing intracytoplasmic sperm injection did not reveal any statistical significance between female BMI and the clinical outcomes. $^{7-12}$

\section{Methods}

This is a prospective cohort research, conducted on 1517 couples who had fresh ICSI cycles of autologous oocytes at Aljazeerah Hospital IVF Unit Kasralainy hospitals, Alazhar university hospitals from February 2016 to March 2018. The couples were involved in fresh ICSI protocol with embryo transfer of day five embryos.

\section{Ethical committee approval}

Patient's enrollment in this study started after approval of Ethical committee of Aljazeerah Hospital.

\section{Consent}

All women who accepted to participate in this study signed an informed consent after complete explanation of the procedure to the 
participant woman.

The cases in the current study were managed by a similar controlled ovarian stimulation protocol, a long protocol.

Transvaginal sonography was performed to assess the follicular growth during controlled ovarian stimulation, and the serum levels of E2, LH, and FSH were measures.

Then 2 high-quality days 5 embryos were transferred. Progesterone injection and dydrogesterone tablets were used for luteal phase support starting from the day of oocyte retrieval.

The serum $\beta$-hCG levels after 14 days of embryo transfer were assayed. A Transvaginal ultrasound examination on day 35 after ET was performed. Clinical pregnancy was diagnosed when a gestation sac and a fetal heartbeat in the uterus were revealed by sonography, and the abdominal ultrasound examination was repeated at $4^{\text {th }}$ gestational month.

1517 cases were prepared for ICSI research cases were categorized according to the corresponding males and females BMI into lean male\& lean females (LM-LF) $(\mathrm{N}=617,40.7 \%)$, lean male\& non-lean female (LM-NLF) $(\mathrm{N}=151,10.0 \%)$, non-lean male\& lean female (NLM-LF) $(\mathrm{N}=562,37.0 \%)$ and non-lean male non-lean female $(\mathrm{N}=187,12.3 \%)$; lean male or female if $\mathrm{BMI}<25.0\left(\mathrm{~kg} / \mathrm{m}^{2}\right)$ otherwise is considered non-lean.

\section{Statistical methods}

The collected data were coded, tabulated, and statistically analyzed using IBM SPSS statistics (Statistical Package for Social Sciences) software version 18.0, IBM Corp., Chicago, USA, 2009.Descriptive statistics were done for quantitative data as minimum\& maximum of the range as well as mean $\pm \mathrm{SD}$ (standard deviation) for quantitative data, median, while it was done for qualitative data as number and percentage.

Inferential analyses were done for quantitative variables using independent t-test in cases of two independent groups, ANOVA test for more than two independent groups with post hoc Tuky's test. In qualitative data, inferential analyses for independent variables were done using Chi square test for differences between proportions and Fisher's exact test for variables with small expected numbers. Logistic regression for factors affecting clinical and completed pregnancy among the studied cases. The level of significance was taken at $\mathrm{P}$ value $<0.050$ is significant, otherwise is non-significant.

The sample size in the present study was based on that this is a single group cohort study that included 1517 women who were prepared for ICSI collected from a Aljazeerah hospital IVF center in the period from February 2016 till March 2018 and gave birth to 858 neonates, the neonates were classified according to the corresponding males and females (parents) BMI into lean male\& lean females (LM-
LF) $(\mathrm{N}=318,44.3 \%)$, lean male\& non-lean female (LM-NLF) $(\mathrm{N}=74$, $8.6 \%)$, non-lean male\& lean female (NLM-LF) $(\mathrm{N}=319,37.2 \%)$ and non-lean male non-lean female ( $\mathrm{N}=85,9.9 \%)$, so sub grouping numbers were by chance according to their proportion in the single group cohort who were seeking ICSI and fulfilling the criteria and enrolled in the study in the period from February 2016 till March 2018

The collected data were coded, tabulated, and statistically analyzed using IBM SPSS statistics (Statistical Package for Social Sciences) software version 18.0, IBM Corp., Chicago, USA, 2009.

Descriptive statistics were done for quantitative data as minimum \& maximum of the range as well as mean $\pm \mathrm{SD}$ (standard deviation) for quantitative data, median, while it was done for qualitative data as number and percentage.

Inferential analyses were done for quantitative variables using independent t-test in cases of two independent groups, ANOVA test for more than two independent groups with post hoc Tuky's test. In qualitative data, inferential analyses for independent variables were done using Chi square test for differences between proportions with post hoc Bonferroni test. Logistic and linear regression for factors affecting neonatal outcomes. The level of significance was taken at $\mathrm{P}$ value $<0.050$ is significant, otherwise is non-significant.

In the current work 1517 women were prepared for ICSI collected from Aljazeera hospital IVF center for 2 years and gave birth to 858 neonates, the neonates were classified according to the corresponding males and females (parents) BMI into lean male\& lean females (LMLF) $(\mathrm{N}=318,44.3 \%)$, lean male\& non-lean female (LM-NLF) $(\mathrm{N}=74$, $8.6 \%)$, non-lean male\& lean female (NLM-LF) $(\mathrm{N}=319,37.2 \%)$ and non-lean male non-lean female $(\mathrm{N}=85,9.9 \%)$; lean male or female if $\mathrm{BMI}<25.0\left(\mathrm{~kg} / \mathrm{m}^{2}\right)$ otherwise is considered non-lean.

\section{Results}

Mean female age $+/$-SD among LM-LF $=27.9+/-2.3 \mathrm{yrs}$, LMNLF $=29.2+/-2.5, \quad$ NLM-LF $=28.9+/-2.5 \mathrm{yrs}, \quad$ NLM-NLF $=29.3+/-$ 2.3yrs, Mean male age $+/-$ SD among LM-LF $=31.0+/-2.8 \mathrm{yrs}$, LM$\mathrm{NLF}=32.7+/-3.1 \mathrm{yrs}, \mathrm{NLM}-\mathrm{LF}=32.6+/-3.0 \mathrm{yrs}, \mathrm{NLM}-\mathrm{NLF}=32.7+/-2.8$ yrs, Female BMI mean $+/ \mathrm{SD}$ among $\mathrm{LM}-\mathrm{LF}=22.4 \pm 0.8 \mathrm{Kg} /$ $\mathrm{m}^{2}, \quad \mathrm{LM}-\mathrm{NLF}=28.1+/-1.0 \mathrm{Kg} / \mathrm{m}^{2}, \quad \mathrm{NLM}-\mathrm{LF}=22.4+/-0.8 \mathrm{Kg} / \mathrm{m}^{2}$, NLM-NLF $=27.9+/ 1.0 \mathrm{Kg} / \mathrm{m}^{2}$, male BMI mean $+/ \mathrm{SD}$ among LM-LF=22.3+/-0.9 $\mathrm{Kg} / \mathrm{m}^{2}, \mathrm{LM}-\mathrm{NLF}=22.2+/-0.9 \quad \mathrm{Kg} / \mathrm{m}^{2}$,NLM$\mathrm{LF}=27.8+/-1.0 \mathrm{Kg} / \mathrm{m}^{2}, \mathrm{NLM}-\mathrm{NLF}=27.9+/-0.8 \mathrm{Kg} / \mathrm{m}^{2}$.

Mean female age $+/$-SD among total cohort $=28.5 \pm 2.4$ years

Mean male age $+/$-SD among total cohort $=31.9 \pm 3.0$ years

Female BMI mean $+/$ SD among total cohort $=23.4 \pm 2.3 \mathrm{Kg} / \mathrm{m}^{2}$

Male BMI mean $+/ \mathrm{SD}$ among total cohort $=24.9 \pm 2.9 \mathrm{Kg} / \mathrm{m}^{2}$ (Figure 1) (Table 1).

Figure I A figure shows the number of subgroups involved regarding BMI.

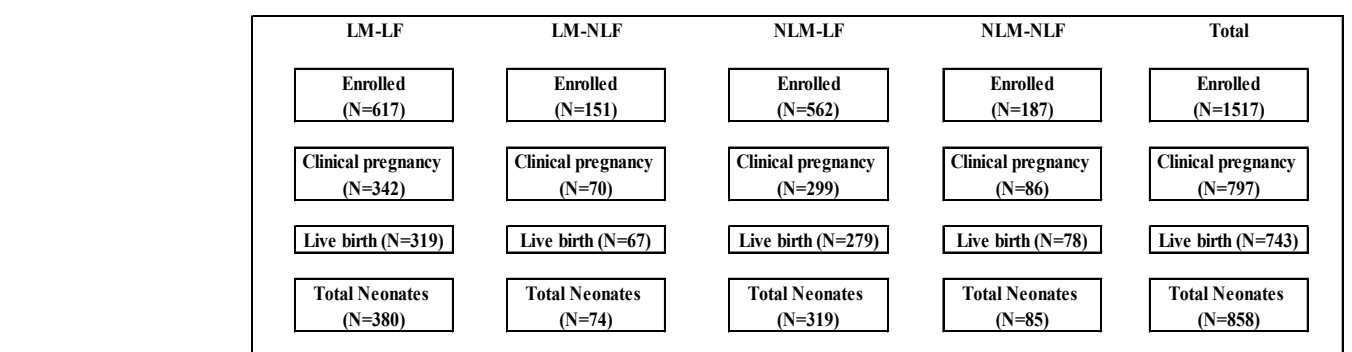

Abbreviations: LM-LF, Lean Male - Lean Female); LM-NLF, Lean Male - Non lean Female; NLM-LF, Non lean Male - Lean Female; NLM-NLF, Non lean Male Non lean Female.

Basic characteristics: This were statistically significant among the BMI subgroups; Female and male ages were significantly lowest among

Citation: Alalfy M, Elgazzar A,Abdelfattah O, et al. The role of body mass index of husband and wife on the neonatal BMI and clinical outcome in ICSI . Obstet Gynecol Int J . 2018;9(5):329-333. DOI: I0.15406/ogij.2018.09.00358 
LM-FL group with no significant difference between other groups Table I Basic characteristics of neonates' mothers regarding such characteristics ( $\mathrm{p}$ value $<0.001$ ) (Table 2 ).

\begin{tabular}{lllllll}
\hline Variables & LM-LF & LM-NLF & $\mathbf{N L M - L F}$ & $\begin{array}{l}\text { NLM-NLF } \\
(\mathbf{N}=85)\end{array}$ & $\begin{array}{l}\text { Total } \\
(\mathbf{N}=858)\end{array}$ & $\mathbf{P}$ \\
\hline Female age (years) & $27.9 \pm 2.3 \mathrm{a}$ & $29.2 \pm 2.5 \mathrm{~b}$ & $28.9 \pm 2.5 \mathrm{~b}$ & $29.3 \pm 2.3 \mathrm{~b}$ & $28.5 \pm 2.4$ & $\wedge<0.00 I^{*}$ \\
Male age (years) & $31.0 \pm 2.8 \mathrm{a}$ & $32.7 \pm 3.1 \mathrm{~b}$ & $32.6 \pm 3.0 \mathrm{~b}$ & $32.7 \pm 2.8 \mathrm{~b}$ & $31.9 \pm 3.0$ & $\wedge<0.00 I^{*}$ \\
Female BMI $\left(\mathrm{Kg} / \mathrm{m}^{2}\right)$ & $22.4 \pm 0.8 \mathrm{a}$ & $28.1 \pm 1.0 \mathrm{~b}$ & $22.4 \pm 0.8 \mathrm{a}$ & $27.8 \pm 1.0 \mathrm{~b}$ & $23.4 \pm 2.3$ & $\wedge<0.00 I^{*}$ \\
Male BMI $\left(\mathrm{Kg} / \mathrm{m}^{2}\right)$ & $22.3 \pm 0.9 \mathrm{a}$ & $22.2 \pm 0.9 \mathrm{~b}$ & $27.8 \pm 1.0 \mathrm{a}$ & $27.9 \pm 0.8 \mathrm{~b}$ & $24.9 \pm 2.9$ & $\wedge<0.00 I^{*}$ \\
\hline
\end{tabular}

^ANOVA test with post hoc Tukey test, Homogenous groups has the same letter a,b, *Significant

Table 2 Neonates characteristics

\begin{tabular}{|c|c|c|c|c|c|c|}
\hline Variables & $\begin{array}{l}\text { LM-LF } \\
(\mathrm{N}=\mathbf{3 8 0})\end{array}$ & $\begin{array}{l}\text { LM-NLF } \\
(\mathrm{N}=74)\end{array}$ & $\begin{array}{l}\text { NLM-LF } \\
(\mathrm{N}=3 \text { |9) }\end{array}$ & $\begin{array}{l}\text { NLM-NLF } \\
(\mathrm{N}=85)\end{array}$ & $\begin{array}{l}\text { Total } \\
(\mathrm{N}=858)\end{array}$ & $\mathbf{P}$ \\
\hline Multiple pregnancy & II (29.5\%)a & $14(18.9 \%) b$ & 74 (23.2\%)a & $14(16.5 \%) b$ & $214(24.9 \%)$ & $\# 0.025 *$ \\
\hline Gestational age at delivery (weeks) & $37.1 \pm 1.9 \mathrm{a}$ & $36.7 \pm 2.3 b$ & $37.2 \pm 1.9 \mathrm{a}$ & $36.5 \pm 2.3 b$ & $37.1 \pm 2.0$ & $\wedge 0.007^{*}$ \\
\hline Prematurity & $102(26.8 \%) \mathrm{a}$ & $34(45.9 \%) b$ & 79 (24.8\%)a & 40 (47.1\%)b & $255(29.7 \%)$ & $\#<0.00$ I* \\
\hline Birth weight $(\mathrm{kg})$ & $2.7 \pm 0.4 \mathrm{a}$ & $2.9 \pm 0.3 b$ & $2.7 \pm 0.4 a$ & $2.9 \pm 0.3 b$ & $2.7 \pm 0.4$ & $\wedge<0.001 *$ \\
\hline Respiratory distress syndrome (RDS) & $32(8.4 \%)$ & II (I4.9\%) & $29(9.1 \%)$ & 10 (II.8\%) & $82(9.6 \%)$ & $\# 0.316$ \\
\hline NICU admission & 51 (I4.6\%) & $16(24.2 \%)$ & 45 (I5.2\%) & $17(23.9 \%)$ & 129 (15.0\%) & $\# 0.162$ \\
\hline
\end{tabular}

^ANOVA test with post hoc Tukey test, \#Chi square test with post hoc Bonferroni test, Homogenous groups has the same letter a,b, *Significant

Multiple pregnancy: This was statistically significantly different among the BMI subgroups; was highest in lean subgroups with no significant

difference among lean subgroups and among non-lean subgroups ( $\mathrm{p}$ value 0.025$)$ in which $\mathrm{LM}-\mathrm{LF}=112$ Cases, $(29.5 \%), \mathrm{LM}-\mathrm{NLF}=14$ Cases, $(18.9 \%)$, NLM-LF=74Cases, $(23.2 \%), N L M-N L F=14$ Cases, $(16.5 \%)$, total number in the cohort of cases $=214$ cases $(24.9 \%)$.

Gestational age at delivery: This was statistically significantly different among the BMI subgroups; was highest in lean subgroups with no significant difference among lean subgroups and among nonlean subgroups. $(\mathrm{p}$ value $=0.007$ ) in which mean gestational age $+/$ SD among $\mathrm{LM}-\mathrm{LF}=37.1 \pm 1.9$ weeks, $\mathrm{LM}-\mathrm{NLF}=36.7 \pm 2.3$ weeks, $\mathrm{NLM}-\mathrm{LF}=37.2 \pm 1.9$ weeks, NLM-NLF=36.5 \pm 2.3 weeks, in total cohort $=37.1 \pm 2.0$ weeks.

Prematurity: Prematurity was statistically significantly different among the BMI subgroups; was lowest in lean subgroups with no significant difference among lean subgroups and among non-lean subgroups. ( $\mathrm{P}$ value $<0.001$ ) in which $\mathrm{LM}-\mathrm{LF}=102$ cases $(26.8 \%)$, LM-NLF $=34$ cases $(45.9 \%)$, NLM-LF $=79$ cases $(24.8 \%)$, NLM$\mathrm{NLF}=40$ cases $(47.1 \%)$, in total cohort $=255$ cases $(29.7 \%)$.

Birth weight was statistically significantly different among the BMI subgroups; was lowest in lean subgroups with no significant difference among lean subgroups and among non-lean subgroups. ( $\mathrm{P}$ value $<0.001$ ) in which mean $+/-$ SD among $\mathrm{LM}-\mathrm{LF}=2.7 \pm 0.4 \mathrm{Kg}$, LM$\mathrm{NLF}=2.9 \pm 0.3 \mathrm{Kg}, \mathrm{NLM}-\mathrm{LF}=2.7 \pm 0.4 \mathrm{Kg}, \mathrm{NLM}-\mathrm{NLF}=2.9 \pm 0.3 \mathrm{Kg}$, in total cohort $=2.7 \pm 0.4 \mathrm{Kg}$

RDS and NICU were lowest in lean subgroups with no statistical significant difference between groups. ( $\mathrm{p}$ value $=0.316,0.162$ consecutively) as regards RDS in which $\mathrm{LM}-\mathrm{LF}=32$ cases $(8.4 \%)$ ,LM-NLF $=11$ cases $(14.9 \%)$, NLM-LF $=29$ cases $(9.1 \%)$, NLMNLF $=10$ cases $(11.8 \%)$, total cohort $=82$ cases $(9.6 \%)$, as regards NICU admission in which LM-LF=51 cases $(14.6 \%), \mathrm{LM}-\mathrm{NLF}=16$ cases $(24.2 \%), \quad N L M-L F=45$ cases $(15.2 \%), \quad N L M-N L F=17$ cases
$(23.9 \%)$, total cohort=129 cases $(15.0 \%)$.

By studying different factors by linear regression only female BMI and GA at delivery ( $p$ value $<0.001$ ) had significant increasing effects, while pregnancy number had statistically significant decreasing effects on neonatal birth weight. ( $p$ value $<0.001$ ). As regards Female BMI $\left(\mathrm{kg} / \mathrm{m}^{2}\right)$ Regression coefficient $=0.040$,Standard error $=0.002,95 \%$ Confidence interval $=0.037-0.044$, As regards Male BMI $\left(\mathrm{kg} / \mathrm{m}^{2}\right)$ Regression coefficient $=-0.002$, Standard error $=0.001$, $95 \%$ Confidence interval $=-0.004-0.001$, as regards gestational age (weeks) Regression coefficient $=0.137$, Standard error $=0.002$, $95 \%$ Confidence interval $=0.132-0.141$, concerning pregnancy number Regression coefficient $=-0.171$, Standard error $=0.008,95 \%$ Confidence interval $=-0.186--0.156$ (Table 3 ).

Table 3 Linear regression for factors affecting neonatal birth weight

\begin{tabular}{lllll}
\hline Variables & $\boldsymbol{\beta}$ & $\mathbf{S E}$ & $\mathbf{P}$ & $\mathbf{9 5 \%} \mathbf{C l}$ \\
\hline Constant & -3.027 & 0.106 & $<0.00 \mathrm{I} *$ & $-3.236--2.8 \mathrm{I} 8$ \\
Female BMI $\left(\mathrm{kg} / \mathrm{m}^{2}\right)$ & 0.04 & 0.002 & $<0.00 \mathrm{I} *$ & $0.037-0.044$ \\
Male BMI $\left(\mathrm{kg} / \mathrm{m}^{2}\right)$ & -0.002 & $0.00 \mathrm{I}$ & 0.124 & $-0.004-0.00 \mathrm{I}$ \\
GA (weeks) & 0.137 & 0.002 & $<0.00 \mathrm{I} *$ & $0.132-0.14 \mathrm{I}$ \\
Pregnancy number & $-0.17 \mathrm{I}$ & 0.008 & $<0.00 I^{*}$ & $-0.186-0.156$
\end{tabular}

Total=858, $\beta$, Regression coefficient; SE, Standard error; $\mathrm{Cl}$, Confidence interval, *significant, R2=0.91 I

By studying different factors by logistic regression only female obesity and multiple pregnancies had significant increasing effects on prematurity. ( $\mathrm{p}$ value $<0.001$ ), in which Female obesity Regression coefficient $=1.839$, Standard error $=0.234$, Odds ratio $=6.288$ : Confidence interval $=3.974-9.949$, concerning male 
obesity Regression coefficient $=0.295$, Standard error $=0.196$, Odds ratio $=1.34395 \%$ Confidence interval $=0.914-1.973$, Multiple pregnancy Regression coefficient $=3.227$, Standard error $=0.217$, Odds ratio $=25.212$ Confidence interval $=16.475-38.580$ (Table 4$)$.

Table 4 Logistic regression for factors affecting prematurity

\begin{tabular}{lllll}
\hline Variables & $\boldsymbol{\beta}$ & SE & $\mathbf{P}$ & OR $(95 \% \mathbf{C I})$ \\
\hline Female obesity & 1.839 & 0.234 & $<0.00 I^{*}$ & $6.288(3.974-9.949)$ \\
$\begin{array}{l}\text { Male obesity } \\
\begin{array}{l}\text { Multiple } \\
\text { pregnancy }\end{array}\end{array}$ & 0.295 & 0.196 & 0.134 & $1.343(0.914-1.973)$ \\
\hline
\end{tabular}

Total=858, $\beta$, Regression coefficient; SE, Standard error; OR, Odds ratio; $\mathrm{Cl}$, Confidence interval, *significant

By studying different factors by logistic regression only female obesity and multiple pregnancy had significant increasing effects ( $p$ values $=0.031,<0.001$, consecutively) while GA had significant decreasing effect on RDS. ( $p$ value $<0.001$ ). As regards female obesity Regression coefficient $=0.611$, Standard error $=0.283$, Odds ratio $=1.842,95 \%$ Confidence interval $=1.058-3.208$, concerinig male obesity Regression coefficient $=0.127$, Standard error $=0.243$, Odds ratio $=1.136,95 \%$ Confidence interval $=0.705-1.828$, as regards gestational age (weeks) Regression coefficient $=-0.081$, Standard error $=0.006$, Odds ratio $=0.922$, Confidence interval $=0.911-$ 0.933 ,multiple pregnancy, Regression coefficient $=1.361$, Standard error $=0.235$, Odds ratio $=3.899$ Confidence interval $=2.461-6.176$ (Table 5).

Table 5 Logistic regression for factors affecting RDS

\begin{tabular}{lllll}
\hline Variables & $\boldsymbol{\beta}$ & SE & $\mathbf{P}$ & OR $(95 \% \mathrm{CI})$ \\
\hline Female obesity & $0.6 \mathrm{II}$ & 0.283 & $0.03 \mathrm{I}^{*}$ & $\mathrm{I} .842(\mathrm{I} .058-3.208)$ \\
Male obesity & 0.127 & 0.243 & 0.601 & $\mathrm{I} .136(0.705-1.828)$ \\
GA (weeks) & $-0.08 \mathrm{I}$ & 0.006 & $<0.00 \mathrm{I} *$ & $0.922(0.9 \mathrm{II}-0.933)$ \\
$\begin{array}{l}\text { Multiple } \\
\text { pregnancy }\end{array}$ & $\mathrm{I} .36 \mathrm{I}$ & 0.235 & $<0.00 \mathrm{I} *$ & $3.899(2.46 \mathrm{I}-6.176)$ \\
\hline
\end{tabular}

Total=858, $\beta$, Regression coefficient; SE, Standard error; OR, Odds ratio; $\mathrm{Cl}$, Confidence interval, *significant

By studying different factors by logistic regression only female obesity and multiple pregnancy had significant increasing effects ( $p$ values $=0.011,<0.001$ consecutively) while GA had significant decreasing effect on NICU admission. ( $p$ value $<0.001$ ) as regards female obesity Regression coefficient $=0.584$, Standard error $=0.231$, Odds ratio $=1.794$,Confidence interval $=1.142-2.819$, concerning male obesity Regression coefficient $=0.107$, Standard error $=0.197$, Odds ratio $=1.113,95 \%$ Confidence interval $=0.757-1.637$, gestational age (weeks) Regression coefficient $=-0.060$, Standard error $=0.005$, Odds ratio $=0.942$, Confidence interval $=0.933-0.950$, multiple pregnancy: Regression coefficient $=0.904$, Standard error $=0.197$, Odds ratio $=2.469$, Confidence interval $=1.678-3.633$ (Table 6).

Table 6 Logistic regression for factors affecting NICU admission

$\begin{array}{lllll}\text { Variables } & \beta & \text { SE } & P & \text { OR }(95 \% \mathrm{CI})\end{array}$

\begin{tabular}{lllll}
\hline Female obesity & 0.584 & $0.23 \mathrm{I}$ & $0.0 \mathrm{I} \mathrm{I}^{*}$ & $\mathrm{I} .794(\mathrm{I} .142-2.8 \mathrm{I})$ \\
Male obesity & 0.107 & 0.197 & 0.586 & $\mathrm{I} .113(0.757-\mathrm{I} .637)$ \\
GA (weeks) & -0.06 & 0.005 & $<0.00 I^{*}$ & $0.942(0.933-0.950)$ \\
$\begin{array}{l}\text { Multiple } \\
\text { pregnancy }\end{array}$ & 0.904 & 0.197 & $<0.00 I^{*}$ & $2.469(\mathrm{I} .678-3.633)$ \\
\hline
\end{tabular}

Total $=858, \beta$, Regression coefficient; SE, Standard error; OR, Odds ratio; $\mathrm{Cl}$, Confidence interval, *significant.

\section{Discussion}

A previous similar study that was conducted in china with the aim to investigate the integrated impact of Chinese couples' body mass indices on the clinical outcomes of IVF or intracytoplasmic sperm injection and the clinical characteristics of their neonates, involving neonatal BMI, in study involved a total of 12061 first fresh IVF/ICSI cycles with autologous oocytes in 2014 ,The main outcome in their research was live birth rate, and for the neonates was neonatal birth weight .they found that in IVF cycles, couples with a female BMI $>=25 \mathrm{~kg} / \mathrm{m} 2$ had a statistically significant higher odds of miscarriage and a statistically significant lower odds of live birth than partners with both male and female BMIs $<25 \mathrm{~kg} / \mathrm{m} 2$. Also Concerning ICSI cycles, no statistical significant correlation was displayed between the couples' BMIs and the live birth rate. The neonatal birth weight of singletons conceived via IVF/ICSI management cycles was statistically significantly higher when their couples BMIs were greater on the other hand; no statistical significant differences were noticed in the neonatal birth weight of twins conceived via IVF/ICSI management cycles.

The research team of investigators came to the conclusion that raised female BMI negatively impacted live births obtained via IVF. Concerning ICSI, no statistical significant differences were observed in the clinical outcomes regarding parental BMI. The singleton neonates' neonatal birth weights were raised with parental BMI conceived via IVF/ICSI protocols. On the other hand, parental BMI did not statistically significantly influence the neonatal birth weight of twins conceived via IVF/ICSI. ${ }^{10}$

In the present study, Assessment of different factors by linear regression only female BMI and GA at delivery ( $p$ value $<0.001$ ) had significant increasing effects, while pregnancy number had statistically significant decreasing effects on neonatal birth weight ( $p$ value $<0.001$ ). By studying different factors by logistic regression only female obesity and multiple pregnancy had significant increasing effects on prematurity ( $\mathrm{p}$ value $<0.001)$.

While a previous research made revealed that a significantly larger increased risk of neonatal mortality with pre-gravid obesity in IVF pregnancies compared to non-IVF pregnancies. High as well as low BMI before IVF are independent risk factors for many adverse maternal and neonatal outcomes. Therefore, optimal body weight prior to ICI is recommended in order to improve not only ICSI pregnancy rates but also neonatal clinical outcomes in postnatal period. ${ }^{13-16}$

In a previous study revealed that, neonates of obese females who develop RDS more commonly and are at raised risk of neonatal death. However, SGA neonates are more common in females who were underweight before ICSI. The impact of elevated pre-pregnancy BMI on cesarean delivery rates and preeclampsia do not seem to be as extreme influence in ICSI in comparison to non-ICSI pregnancies. 
Rates of RDS were greater in neonates born to mothers with higher BMI in both ICSI and non-ICSI research groups. ${ }^{13-16}$

Moreover, in the present study, By studying different factors by logistic regression only female obesity and multiple pregnancy had significant increasing effects ( $\mathrm{p}$ values $=0.031,<0.001$,consecutively) while GA had significant decreasing effect on RDS ( $p$ value $<0.001$ ). By studying different factors by logistic regression only female obesity and multiple pregnancy had significant increasing effects ( $p$ values $=0.011,<0.001$ consecutively) while GA had significant decreasing effect on NICU admission ( $p$ value $<0.001$ ).

Another research in Bangladesh investigated maternal BMI impact on child health care parameters including neonateal birth weight revealed that overweight and obese women in comparison to normal weight females are more liable to experience gestational complications.. Underweight females were 1.3 times more liable to have children with stunting pattern of growth and 1.6 times more liable to experience wasting in comparison to normal weight women. Maternal BMI was not statistically significantly correlated with increased risk low birth weight despite the fact that in certain cases risk was higher. The research team came to the following conclusions in which elevated levels of maternal overweight and obesity have considerable impact on clinical Outcomes of new borns including birth weight, at the same time as underweight was a risky issue for newborn health. The findings reveal that weight management is crucial to avoid $t$ adverse birth and neonatal health outcomes. ${ }^{17}$

\section{Conclusion}

The present study revealed that neonatal birth weight in ICSI is significantly influenced by raised maternal BMI and gestational age at which delivery occurred.

\section{Acknowledgements}

The authors would like to thank all medical staff members who helped us in completing this research in aljazeerah IVF Centre.

\section{Disclosure statement}

All authors declare that there are no any financial and personal relationships with other people or organizations that could inappropriately influence (bias) their work.

\section{Conflicts of interest}

The author declares that they have no conflict of interest.

\section{References}

1. Talmor A, Dunphy B. Female obesity and infertility. Best Pract Res Clin Obstet Gynaecol. 2015;29(4):498-506.

2. Ozekinci M, Seven A, Olgan S, et al. Does obesity have detrimental effects on IVF treatment outcomes? BMC Womens Health. 2015;15:61.

3. CaillonH, Fréour T, Bach-Ngohou K, et al. Effects of female increased body mass index on in vitro fertilization cycles outcome. Obes Res Clin Pract. 2015;9(4):382-388.

4. Koning AM, Mutsaerts MA, Kuchenbecker WK, et al. Complications and outcome of assisted reproduction technologies in overweight and obese women. Hum Reprod. 2012;27(2):457-467.

5. Moragianni VA, Jones SML, Ryley DA. The effect of body mass index on the outcomes of first assisted reproductive technology cycles. Fertil Steril. 2012;98(1):102-108.

6. Schliep KC, Mumford SL, Ahrens KA, et al. Effect of male and female body mass index on pregnancy and live birth success after in vitro fertilization. Fertil Steril. 2015;103(2):388-395.

7. Edwards MO, Kotecha SJ, Kotecha S. Respiratory distress of the term newborn infant. Paediatr Respir Rev. 2013;14(1):29-36.

8. Lucovnik M, Blickstein I, Verdenik I, et al. Impact of pre-gravid body mass index and body mass index change on preeclampsia and gestational diabetes in singleton and twin pregnancies. J Matern Fetal Neonatal Med. 2014;27(18):1901-1904.

9. WHO. Feto-maternal nutrition and low birth weight. World Health Organization; 2011

10. Wang X, Hao J, Zhang F, et al. Effects of female and male body mass indices on the treatment outcomes and neonatal birth weights associated with in vitro fertilization/intracytoplasmic sperm injection treatment in China. Fertil Steril. 2016;106(2):460-466

11. Provost MP, Acharya KS, Acharya CR, et al. Pregnancy outcomes decline with increasing body mass index: analysis of 239,127 fresh autologous in vitro fertilization cycles from the 2008-2010 Society for Assisted Reproductive Technology registry. Fertil Steril. 2016;105(3):663-669.

12. Ozekinci M, Seven A, Olgan S, et al. Does obesity have detrimental effects on IVF treatment outcomes? BMC Womens Health. 2015;15:61.

13. Singh N, Gupta P, Mittal S, et al. Correlation of body mass index with outcome of in vitro fertilization in a developing country. Arch Gynecol Obstet. 2012;285(1):259-263

14. Mori N, Asakura K, Sasaki S. Differential dietary habits among 570 young underweight Japanese women with and without a desire for thinness: a comparison with normal weight counterparts. Asia Pac J Clin Nutr. 2016;25(1):97-107.

15. Catteau A, Caillon H, Barriere $\mathrm{P}$, et al. Leptin and its potential interest in assisted reproduction cycles. Hum Reprod Update. 2016;22(3):320-341.

16. Lao XQ, Ma W, Chung RY, et al. The diminishing socioeconomic disparity in obesity in a Chinese population with rapid economic development: analysis of serial cross-sectional health survey data 2002-2010. BMC Public Health. 2015;15:1282.

17. Khan MN, Rahman MM, Shariff AA, et al. Maternal undernutrition and excessive body weight and risk of birth and health outcomes. Arch Public Health. 2017;75:12. 